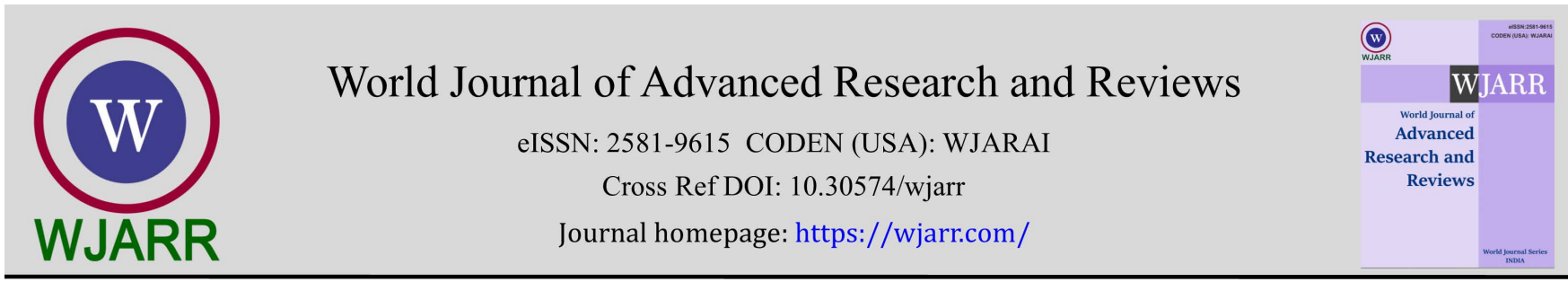

(REVIEW ARTICLE)

Check for updates

\title{
Sustainable ICT: An essential enabler of sustainable research and innovations in Tertiary Institutions in Nigeria
}

\author{
Felix Chukwuma Aguboshim ${ }^{1}$, Ifeyinwa Nkemdilim Obiokafor $2,{ }^{*}$ and Uchenna Paulinus Onwuka ${ }^{3}$ \\ ${ }^{1}$ Department of Computer Science, Federal Polytechnic, Oko Nigeria. \\ 2 Department of Computer Science Technology, Anambra State Polytechnic, Mgbakwu, Nigeria. \\ ${ }^{3}$ Department of Computer Science, Nnamdi Azikiwe University, Awka. Nigeria.
}

World Journal of Advanced Research and Reviews, 2021, 10(01), 125-131

Publication history: Received on 01 March 2021; revised on 02 April 2021; accepted on 04 April 2021

Article DOI: https://doi.org/10.30574/wjarr.2021.10.1.0139

\begin{abstract}
Globally, sustainable Information and Communication Technology (ICT) innovations are significant in realizing sustainable research and innovations in tertiary institutions. Despite this laudable global impact of ICT on research and innovations, poor ICT policies and practices, coupled with bad economy that plagued sustainable ICT innovations in Nigeria have hindered sustainable research and innovations in tertiary institutions. This study adopted the Unified Theory of Acceptance and Use of Technology (UTAUT) as the study conceptual framework. UTAUT model postulates that users' acceptance behaviour toward a technology is determined by users' decision to use a technology, and the perceived benefits thereof. The authors explored a narrative review, analysis, and synthesis of vast works of literature that revealed significant information on impact of sustainable ICT on sustainable research and innovations in Nigeria. The authors also extracted peer-reviewed articles within the last five years from electronic databases using some keywords such as "ICT impact on research innovations", "ICT adoption in tertiary institutions", "ICT usability Trends", and so on. The results from this study revealed that strict adherence to ICT policies, laws, guidelines, and sustainability, coupled with good formulation and communication of same, are the major impact of sustainable ICT that can leverage research and innovations in tertiary institutions in Nigeria. Results from this study may increase understanding, minimize corrupt practices and encourage value for ICTs that can positively impact research and innovations in tertiary institutions for economic development in Nigerian.
\end{abstract}

Keywords: ICT; Research and innovations; Tertiary institutions; Adoption; Sustainability

\section{Introduction}

The term ICT comprised basically, the physical devices and some other infrastructures used for data and information gathering, storage, processing, and the required platform for the transmission and disseminating of same [15] and [16]. Globally, there are a lot of best practices that show how research database repository, which is heavily driven by sustainable ICT, has positively influenced research innovation for tertiary education [5]. The global aim of sustainable ICT in research innovation for tertiary education is to encourage research database repository by leveraging a single version of the truth and a single reference for critical master data, across geographies and educational institution. Sustainable ICT is of significant importance in realizing or attaining sustainable research innovation for national economic growth in Nigeria. Sustainable ICTs are powerful enablers and pedagogical tools for diffusing knowledge and information for research and innovations globally. Sustainable ICT is of concern to any tertiary institution for sustainable research innovation and education.

\footnotetext{
${ }^{*}$ Corresponding author: Ifeyinwa Nkemdilim Obiokafor

Department of Computer Science Technology, Anambra State Polytechnic, Mgbakwu, Nigeria.

Copyright (C) 2021 Author(s) retain the copyright of this article. This article is published under the terms of the Creative Commons Attribution Liscense 4.0.
} 
Research and innovations in tertiary institutions which is the pivot for economic development, has remained relatively low or unnourished in Nigeria due to corrupt and poor ICT policies and practices, coupled with bad economy that plagued sustainable ICT innovations. Sustainable research database repository globally are becoming complex and sophisticated, very expensive, unable to cope with the reality of ever-increasing data complexity, and ever dependable on the implementation of effective and sustainable ICT rules and policies. A significant relationship exists between sustainable ICT and sustainable research database repository and data governance for sustainable research and innovation in tertiary institutions [4]. This is because sustainable ICT makes sure that all rules, policies, roles, responsibilities and tools needed to leverage sustainable research innovation for tertiary education are put in place to ensure that database research repositories are accurate, consistent, complete, available and secure [13].

Tertiary education that is driven by unsustainable ICT with good structured research database repository will fail because sustainable ICT is what defines sustainable research innovations in tertiary education [15], and occupies a significant key position in leveraging sustainable research database repository [16]. Users' value for ICT innovations is measured by users' adoption, acceptance, and sustainability of the technology [11]. It takes sustainable ICT for research innovation in tertiary education to thrive [15]. This is because in all facets of life and technological advancements. ICT plays the role of being the major enabler of sustainable research innovations [20]. Despite the significant importance of ICT technological innovations in Nigeria, its poor adoption, usage, and sustainability, worsened by corrupt practices, has ravaged virtually every system in the nation, especially sustainable research innovation for sustainable tertiary education and national economic growth.

\subsection{Problem Statement}

Sustainable research innovation for sustainable tertiary education is ICT driven and can only be meaningful when it aligns with the ICT policies, rules, goals, and values in a sustainable manner. Our purpose in this study was to identify the challenges of sustainable ICT that negatively impact sustainable research innovation for sustainable tertiary education in Nigeria. The general IT problem was the poor performance or sustainability of research innovation for sustainable tertiary education majorly due to poor adoption, usage, and sustainability of ICT in Nigeria as a result of corrupt ICT practices and policies in Nigeria. The specific IT problem is that some ICT stakeholders in Nigeria formulate, institute and communicate ICT policies, laws, guidelines, and value system that do not favour sustainable research innovations for sustainable tertiary education. This is because such policies and practices are rendered impotent by non-adoption of ICT international standards in all facets of the Nigeria education and legal system for standardized behaviours and values towards sustainable ICT.

\subsection{Research Question}

What are ICT policy standards and value system strategies used by tertiary institution stakeholders to sustain ICT innovations to leverage sustainable research innovation for sustainable tertiary education?

\section{Literature Review}

Sustainable research innovation for sustainable tertiary education is far-fetched in Nigeria because its enabler (ICT) is not adequately valued, adopted or used. This is because it takes sustainable ICT to achieve sustainable research innovation for sustainable tertiary education [14]. Users' value for ICT, its adoption and use can be determined by its sustainability. Similarly, users motivation towards the use of ICT is seen as a function of their perceived level of trust, perceived ease of use and easy-to-use [1]. Therefore, failure to value and use ICTs can undermine even the strongest research innovation plans, because what contributes to non-sustainable ICT innovations has proven to cause or be related to non-sustainable research innovation [18]. Sustainable ICT technological innovations have significant importance in leveraging sustainable research innovation for sustainable tertiary education [4]. It is acknowledged and identified by most researchers that content and functional value that interacts positively with appropriate ICT policies and guidelines that mediate significantly in users are the key determinants of impactful and sustainable technology [1], [14] and [16]. Other determinants of sustainable ICT are perceived value and provision of security facilities that significantly influence its enablement for any sustainable innovation [2].

In Nigeria, it is evident that corrupt policies and practices, ignorance, illiteracy, and bad economy, among others, are the major factors militating against sustainable ICT. This is because standard international ICT policy measures, laws, and infrastructures required to handle ICT sustainability are rather relegated to the background. Unquestioning obedience to ICT rules and policies, coupled with policy enforcement, and enterprise definitions have become attitudinal and are no longer reliable in sustaining ICT innovations. Due to corrupt practices in Nigeria, ICT innovations have been made to operate in line with human attitudes or dispositions. As a result of these attitudinal bewitchment that rendered ICT policies, ethics and values futile and purposeless, and its sustainability impotent. There are empirical research evidences 
that show that while human adherence to required ICT principles and policies are the major links the ICT sustainability, the negligence or none obedience of same impact great risk to the ICT sustainability [15], [16] and [20]. Violations of established ICT policies and safeguards by users especially by some of our so-called honourable men in our society have led to poor sustainable database administration and diversified economy. Two major drivers of ICT acceptance have been identified: perceived usefulness (PU) and perceived ease of use (PEOU) [14] and [18]. Perceived benefit and perceived self-efficacy were found significant to be driver of ICT usage [19]. As stated by [3] and [12], self-efficacy appears to be the most powerful factor influencing ICT users' behavioural intension and trust because users who believe in their ability to effectively use ICT services, are more likely to trust using such technology, as well as being more motivated to adopt it in the future. [3] also found habit and motivation as significant determinants of ICT usage along with behavioural intention.

\subsection{Conceptual Framework}

The Unified Theory of Acceptance and Use of Technology (UTAUT), proposed by Venkatesh, Morris, Davis, \& Davis (2003) were adopted as the conceptual framework for this study. According to UTAUT model, users' acceptance behaviour toward a technology is determined by users' decision to use a technology, and the perceived benefits thereof. The theory considers the following factors as determinants of users' acceptance behaviour toward a technology: (a) user adoption behaviour toward intention to use ICT, and (d) users' usage behaviour of ICT. Explaining further, UTAUT model claimed that both user adoption and usage of ICT are affected by four constructs: performance expectancy (PE), effort expectancy (EE), social influence (SI), and facilitating conditions (FC). User adoption and usage of ICT are also affected by four moderators: gender, age, experience and voluntariness of use. UTAUT model in recent times has been widely adopted [17]. UTAUT was adapted as our theoretical foundation to study how ICT sustainable can impact research and innovations in tertiary institutions in Nigeria

\subsection{Tools for Sustainable Research Innovation for Sustainable Tertiary Education}

Objectives of Sustainable research innovation for sustainable tertiary education programs are accomplished within appropriate ICT tools. Research innovation for sustainable tertiary education might not be a typical IT project but it is certainly driven by appropriate ICT tools. Sustainable research innovation for sustainable tertiary education is exemplified by sustainable ICT policies that are designed to automate research database repository and many intangible activity connected with research innovations in tertiary education, and collect, process and use large data sets of various formats obtained from many sources require for sustainable research innovation for sustainable tertiary education. Tools that provide immediate and very cheap communication protocols, launch new dissemination channels (ecommerce) for sustainable research innovation, and distribute same products as well. ICT is an enabler of most functioning enterprises and societies including sustainable research innovation for sustainable tertiary education. Being an enabler of all facets of life, ICT has changed or affected the way jobs are executed. Today we talk of e-work; erest, e-commerce, e-banking, e- travel, etc. Research and innovations in tertiary institutions are not left out in the list that require ICT platform for its sustainability.

\section{Methodology}

In this study we adopted a narrative review methodology and explicitly explained the methodological commitments of narrative inquiry. We reviewed significant information based on the study conceptual framework, existing systems that enhance ICT sustainability. Prior research findings related to our study were also reviewed, analysed and synthesized. Our research criteria and criteria for inclusion of the relevant prior research findings were explicit in our review process, choice of search keywords and term identification, article identification, quality assessment, data extraction, and data synthesis. Our intention is to draw a holistic interpretations or conclusions based on our reviews. In relation to [9], narrative review is efficient and appropriate where analysis and synthesis of different and related research findings are required to draw holistic interpretations or conclusions alongside with, existing theories, and models. Methodological triangulation, which is the use of multiple sources of data to gain multiple perspectives, maximize reliability and validation of data in order to build coherent justification of data interpretation that relates to the study case or phenomenon was adopted. Methodological triangulation to ensures the reliability and validity of data, and justification of interpretations from the reviews [6].

\subsection{Data Collection}

Findings from literature that are relevant and related to our study were reviewed. Findings majorly came from the ProQuest databases, ScienceDirect, Walden University international library databases and peer-reviewed, and other related texts. We also used phrases and terms as key search words in the databases for related literature on ICT sustainability for sustainable research innovation for sustainable tertiary education in Nigeria. Our reviews 
incorporated 22 references. Ninety six percent (96\%) of total references incorporated in the study is peer-reviewed, while $(67 \%)$ are peer-reviewed journals that are within the last 5 years.

\section{Analysis and Synthesis of Prior Research}

Relevant and wide varieties of important literature have identified ICT as important innovation-enablers of sustainable research innovation for sustainable tertiary education [15]. Despite the implementation of ICT innovations in Nigeria, sustainable research innovations for sustainable tertiary education have remained weak and vulnerable. This is because there are evidences that suggest that corrupt practices and non-adherence to ICT policies and rules are increasingly exploiting ICT sustainability, and adversely affecting research database repository implementation for sustainable research innovation in tertiary education. Reasons for non-sustainable ICT that adversely affected sustainable tertiary education included: (a) problems associated with corrupt policies and poor ICT systems usability [21], (b) not placing required value to ICT by users [8], and (c) limited perception of the usefulness and ease of use of ICT innovations [1].

As mentioned above, the major reasons for this might be corrupt practices that negated international ICT policies, ignorance, or illiteracy. This claim is supported by United Nations Development Programme (UNDP) statistical report that revealed $14 \%$ of Nigerian adults considering corruption as the major significant challenges militating against sustainable ICT in Nigeria [21]. There is a need for awareness and training in the country for stakeholders of tertiary institutions in Nigeria to understand and be able to perceive available technological innovations as useful and easy-touse in relation to sustainable research innovations. This may bring about increase in research innovations in Nigerian tertiary institutions as ICT becomes sustainable in a manner that will leverage economic development and social change.

Other factors that contributed significantly to the challenges of sustainable ICT have been classified into four groups by [7] as (i) management of ICT processes, policies and guidelines, (ii) literacy level of ICT users and how it impacts usage and adoption, (iii) well defined ICT project size, goals, performance, robustness, and implementation, and (iv) technology failures resulting from ICT use and misuse. Contributing, [10] suggested three major strategies to improve or manage ICT adoption, usage, and sustainability such as (i) adherence to usability guidelines and policies, (ii) process control, and (iii) information and data transmission and dissemination.

\section{Discussions}

This paper conducts an evidenced-based study using Web content analysis that substantiates the current state of research innovations in tertiary education in Nigeria. When ICTs are fully implemented in all required sectors and systems, Nigeria may start to reap the dividends of sustainable research innovations and economic growth as witnessed in the developed nations with the best ICT facilities. ICT projects in Nigeria are failing, despite much enthusiasm and optimism and global trend for ICT and its significant importance for sustainable research innovations. The adoption of technology solutions in Nigeria has remained poor or slow due to corrupt practices that are contrary to ICT rules, ethics, and policies [20]. ICT innovations in Nigeria are not making maximum impact when compared with other African countries. These are a few of our observations:

\subsection{The Value Placed on ICT by the Stakeholders Tertiary Institutions in Nigeria.}

The contribution of any technological innovation can only be realized when and if the technology is widely diffused and used. The value placed on any technological innovation is measured by its adoption, acceptance, and sustainability. It is hypocritical to observe that the various arms of the Government make much noise about ICT while in truth they do not value it because they do not see ICT services as a means to sustainable research innovations and economic productivity of the nation. ICT is seen more like a "corruption-exposer" technology.

\subsection{Inadequate and Incomplete Implementation of ICT}

Most technological innovations in Nigeria lack solid and proper implementations. There are virtually no complete or proper implementations, because they often end with the purchase and implementation of the physical IT equipment. Little or no efforts are made to implement the various platforms that drive the IT. What is referred to as ICTs are mere independently operated ITs. There are no "information and communication", aspect of the ICTs to drive the ICT databases if they exist. For instance, traffic lights in Nigeria are not properly implemented and so are not adequately or properly put to use. Nigeria is about the only country where cameras are mounted on the traffic light with no corresponding machinery to adequately capture, document and book or fine offenders. It is not possible to run ICT without proper database documentation, and effective communication platforms to drive the database. How can one 
run an automated traffic system without proper databases, data governance and documentation of vehicles and their owners.

It is a common phenomenon in Nigeria to observe policemen and other touts being used to check traffic light offenders, when the traffic lights are supposedly embedded or supposed to be embedded with monitoring cameras. What a waste of resources. The same is applicable to other establishments such as NRSC, Police, Ministry of Justice, Tertiary Institutions, etc. Our ICTs are not adequately implemented or used. Therefore its sustainability is crossly endangered, especially now that ICT innovations are widely integrated into ambient or ubiquitous environments. The nonsustainability of ICT in Nigeria is the major reason for low research innovation growth in Nigeria.

\subsection{Corrupt Practices and Policies}

Corruption appears to be the major hindrances to sustainable ICT for maximum research innovation growth in Nigeria. Technology does not have an attitude or designed to exhibit attitudinal or emotional behaviours. ICTs are not designed to have emotions, behaviours, and thoughts, but to operate according to the specified programs and protocols. When ICT innovations are subjected and corrupted with human emotions, behaviours, and thoughts, they become useless, unimpactful and their outputs nonsensical including sustainable research innovation that it intended to leverage. Some instances: (a) the so-called honourable men in the society breaking the traffic light. (a) Using the exit door for an entrance. (c) Using their vehicles with government registration numbers even when they have left the office, and (d) Some Nigerians, because they in the police, army, and others, you do not need to renew their vehicle particulars because the so-called ICT in place has been subjected to have attitudinal behaviour. This is a big slap on the face of technology in Nigeria. This is the major hindrances of sustainable ICT for sustainable development in Nigeria. Embracing technological innovations in Nigeria would mean embracing transparency, which exposes corruption and enforces sustainable development.

\subsection{The Way Forward}

ICT innovations are now handled with better interconnected and interdependent facilities because connectivity is widely integrated into ambient or ubiquitous environments through an intuitive interface or "smart" interaction. The way forward to overcome the hindrances of sustainable research innovation for tertiary education is to ensure the effectiveness in the implementation of sustainable ICTs. The following are recommended: (a) ICT and research database repository stakeholders in tertiary institutions in Nigeria must recognize and understand the significant importance of sustainable ICT to sustainable development. (b) Existing ICT infrastructures, its usability, procurement and implementation for research innovation must be reviewed, and a new system designed to meet the required standards for sustainable tertiary education. (c) In all facets of the Nigeria economy, ICT adoption must follow ICT international standards for standardized behaviours and values towards sustainable ICT. (d) To fully actualize a sustainable ICT that will effectively leverage sustainable research innovation, all stakeholders, irrespective of rank or status should learn to respect the law rather than the persons. ICT innovations, sustainable research innovation in particular, are being slaughtered on the platform of corruption and lawlessness, with little or no regards for intellectual property laws because research database repository are not in place. All research works, projects and seminars in our tertiary institution should be published and stored in standard database repositories, and accessible internationally.

\section{Conclusion}

This study revealed that most technological innovations in Nigeria lack solid and proper implementations due to corrupt practices that fail to align with ICT international standards, ethics, and values required for sustainable ICT. Corrupt practices appear to be the major hindrances to sustainable ICT for sustainable research innovation growth in Nigeria. The findings from this study may encourage social change as more ICT stakeholders in Nigeria learn to adopt international standards for standardized behaviours and values towards sustainable ICT that may improve user morale, preference, attraction, productivity, and increase usability and implementation of research database repository for sustainable research innovations in Nigeria.

\section{Compliance with ethical standards}

\section{Acknowledgments}

My sincere appreciation and thanks to Dr. Felix. Chukwuma. Aguboshim for his wonderful contributions. 


\section{Disclosure of conflict of interest}

There are no conflicts of interest.

\section{References}

[1] Aguboshim FC, Miles GS. Engaging pictorial images and voice prompts interface design strategy to create easy to use banking ATM system interfaces in Nigeria. Journal of Information Engineering and Application (JIEA). 2019; 8(1): 1-14.

[2] Aguboshim FC, Udobi JI. Security issues with mobile IT: A Narrative Review of Bring -Your-Own-Device (BYOD). Journal of Information Engineering and Application (JIEA). 2019; 9(1): 56-66.

[3] Alalwan AA, Dwivedi YK, Rana NP, Lal B, Williams MD. Consumer adoption of Internet banking in Jordan: Examining the role of hedonic motivation, habit, self-efficacy and trust. Journal of Financial Services Marketing. 2015; 20(2): 145-157.

[4] Bennett S. What is information governance and how does it differ from data governance? Governance Directions. 2017; 69(8): 462-467.

[5] Domenico C. Literature analysis on determinant factors and the impact of ICT in Small-Medium Enterprises (SMEs), Procedia-Social and Behavioral Sciences. 2012; 62(1): 93 - 97.

[6] Durif-Bruckert C, Roux P, Morelle M, Mignotte H, Faure C, Moumjid-Ferdjaoui N. Shared decision-making in medical encounters regarding breast cancer treatment: the contribution of methodological triangulation. European Journal of Cancer Care. 2014; 24(4): 461-472.

[7] Dwivedi Y, Wastell D, Laumer S, Henriksen HZ, Myers MD, Bunker D, Elbanna A, Ravishankar MN, Srivastava SC. Research on information systems failures and successes: Status update and future directions. Information Systems Frontiers. 2015; 17(1): 143-157.

[8] GreavuSerban V, Serban O. Social Engineering a General Approach. Informatica Economica. 2014; 18(2): 5-14.

[9] Hill C, Burrows G. New voices: The usefulness of a narrative approach to social work research. Qualitative Social Work: Research and Practice. 2017; 16(2): 273-288.

[10] Ho L, Hsu M, Yen T. Identifying core control items of information security management and improvement strategies by applying fuzzy DEMATEL. Information and Computer Security. 2014; 23(2): 161-177.

[11] Hoffman DD, Singh M, Prakash C. The Interface Theory of Perception Psychonomic. Bulletin \& Review. 2015; 22(6): 1480-1506.

[12] Kelly H. A path analysis of educator perceptions of open educational resources using the technology acceptance model. International Review of Research in Open and Distance Learning. 2015; 15(2).

[13] Koltay T. Data governance, data literacy and the management of data quality. International Federation of Library Associations and Institutions. 2016; 42(4): 303-312.

[14] Marangunic N, Granic A. Technology acceptance model: A literature review from 1986 to 2013. Universal Access in the Information Society. 2015; 14(1): 81-95.

[15] Oladimeji TT, Foltyn GB. ICT and Its Impact on National Development In Nigeria: An Overview. Research \& Reviews: Journal of Engineering and Technology. 2018; 7(1): 1-10.

[16] Olise FP. Information and Communication Technologies (ICTs) and Sustainable Development in Africa: Mainstreaming the Millennium Development Goals (MDGs) into Nigeria's Development Agenda. J SocSci. 2010; 24(3): 155-167.

[17] Oye ND, Alahad N, Abrahim N. The history of UTAUT model and its impact on ICT acceptance and usage by academicians. Education and Information Technologies. 2014; 19(1): 251-270.

[18] Peiris PM, Kulkarni D, Mawatha C. Implications of trust and usability on e-commerce adoption. International Journal of Business and Information. 2015; 10(4): 519-556.

[19] Teoh WM, Siong CC, Lin B, Jiat WC. Factors affecting consumers' perception of electronic payment: an empirical analysis. Internet Research. 2013; 23(4): 465-485. 
[20] Toyo OD, Ejedafiru FE. Utilization of Information and Communication Technologies (ICTs) for Sustainable Economic Development in Nigerian. International Journal of Ergonomics and Human Factors. 2016; 12(2): 2234.

[21] United Nations Development Programme (UNDP), Nigeria 2016 Annual Report Compiled and edited by Lucky Musonda. UNDP Nigeria 2016 Annual Report_web.pdf. 2016.

[22] Venkatesh Morris, Davis Davis. User Acceptance of Information Technology: Toward a Unified View. MIS Quarterly. 2003; 27(3): 425. 